\title{
Correction to: Gamma-ray flux measurement and geotechnical studies at the selected site for the LABChico underground laboratory
}

\author{
A. Aguilar-Arevalo ${ }^{1}$, X. Bertou ${ }^{2}$, C. Canet ${ }^{3}$, M. A. Cruz $^{3}$, A. Deisting ${ }^{4}$, A. Dias ${ }^{4}$, J. C. D'Olivo ${ }^{1}$, F. Favela-Pérez ${ }^{1,2}$, \\ E. A. Garcés ${ }^{5,9, a}$, E. González García ${ }^{6}$, A. González Muñoz ${ }^{5}$, J. O. Guerra-Pulido ${ }^{1}$, J. Mancera-Alejandrez ${ }^{6}$, \\ D. J. Marín-Lámbarri ${ }^{1,5, b}{ }_{1}$, A. M. Martínez Mendoza ${ }^{5, c}$, M. Martínez Montero ${ }^{1}$, J. Monroe ${ }^{4}$, S. Paling ${ }^{7}$, S. Peeters ${ }^{8}$, \\ P. R. Scovell ${ }^{7}$, C. Türkoğlu ${ }^{8}$, I. G. Vallejo Castillo ${ }^{6}$, E. Vázquez-Jáuregui ${ }^{5, \mathrm{~d}}$, J. Walding ${ }^{4}$ \\ ${ }_{1}^{1}$ Instituto de Ciencias Nucleares, Universidad Nacional Autónoma de México, Ciudad de México, México \\ 2 Centro Atómico Bariloche, CNEA/CONICET/IB, Bariloche, Argentina \\ ${ }^{3}$ Centro de Ciencias de la Atmósfera, Universidad Nacional Autónoma de México, Ciudad de México 04510, México \\ ${ }^{4}$ Royal Holloway, University of London, Egham Hill, United Kingdom \\ 5 Instituto de Física, Universidad Nacional Autónoma de México, Ciudad de México 01000, México \\ ${ }^{6}$ Facultad de Ingeniería, Universidad Nacional Autónoma de México, Ciudad de México, México \\ 7 Boulby Underground Laboratory, Boulby Mine, Saltburn-by-the-Sea, United Kingdom \\ 8 Department of Physics and Astronomy, University of Sussex, Brighton, United Kingdom \\ 9 Present Address: Departamento de Física, FES-Cuautitlán, Universidad Nacional Autónoma de México, C.P. 54770 Estado de México, México
}

(c) The Author(s), under exclusive licence to Società Italiana di Fisica and Springer-Verlag GmbH Germany, part of Springer Nature 2022

Correction to: Eur. Phys. J. Plus (2022) 137:210 https://doi.org/10.1140/epjp/s13360-022-02407-1

In this article, the affiliation 'Instituto de Ciencias Nucleares, Universidad Nacional Autónoma de México, Ciudad de México, México' for D. J. Marín-Lámbarri was missing.

Affiliations 3 and 5 have been corrected:

3 Centro de Ciencias de la Atmósfera, Universidad Nacional Autónoma de México, Ciudad de México, 04510, México

5 Instituto de Física, Universidad Nacional Autónoma de México, Ciudad de México, 01000, México

The original article has been revised.

The original article can be found online at https://doi.org/10.1140/epjp/s13360-022-02407-1.

\footnotetext{
a e-mail: egarces@ fisica.unam.mx

b e-mail: marinlambarri@gmail.com (corresponding author)

c e-mail: miichmtz@ciencias.unam.mx

de-mail: ericvj@fisica.unam.mx
} 